\title{
Energy saving in cooperative transmission using opportunistic protocol in MANET
}

\author{
K. Vanisree ${ }^{1}$, V. S. K. Reddy ${ }^{2}$ \\ ${ }^{I}$ (Ece Holy Mary Institute of Technology \&Science, College Bogaram India) \\ ${ }^{2}$ (ECE,Malla Reddy College of Engineering and Technology, India)
}

\begin{abstract}
In this thesis, we study the joint problems of cooperative link and diversity in A Mobile Ad-Hoc Network (MANET) with variable wireless channels. In MANET the wireless nodes are in group and infrastructure less in nature. The major problems faced by wireless communication in real time environment are that of interference and un-reliable communication links. Much research work has been done to overcome this by using various techniques. Cooperative communication and transmission side diversity in the network are the two of the techniques that help in reducing interference and communication link failures. We have also proposed a new technique to find the optimum route as a joint problem of the transmission power at the physical layer and the link selection at the network layer that incurs the minimum cost in terms of energy, no. of hops, available bandwidth and link quality (SNR), outage probability. Analytical results show that our cooperative transmission schemes (OMCTSP) achieves average energy saving of more than $80 \%$ than direct transmission.
\end{abstract}

Keywords: cooperative transmission, , channel gain, diversity, , linkcost minimum energy routing, outage diversity, Variable wireless channels.

\section{Introduction}

A MANET is a multi-hop wireless network that is formed dynamically without infrastructure support. In this thesis we study the joint problems of energy efficient and diversity in wireless ad -hoc network. In wireless network the energy is spend by nodes on communication [1]. First ,Nodes are small in size and typically battery powered, inefficient use of power causes network life time is decreased [2][3]. So past several years most attention in energy-efficient communication in ad hoc networks. The problem is approached in two different ways. At the network layer to find the energy efficient routes selection and at the physical layer energy efficient for wireless channel is called cooperative communication [5],[6].

\subsection{Multi-Hop Relay Model}

The propagation of electromagnetic signals through space, the energy required to establish a link between two nodes are proportional to distance between nodes which are in communication set is raised to fixed exponent is called path loss is assumed to be between 2 or 4.It is advantages, in terms of energy saving through multi-hop routing in adhoc network. The multi-hop network extends coverage range.Figure 1.1 shows the multihop route between nodes

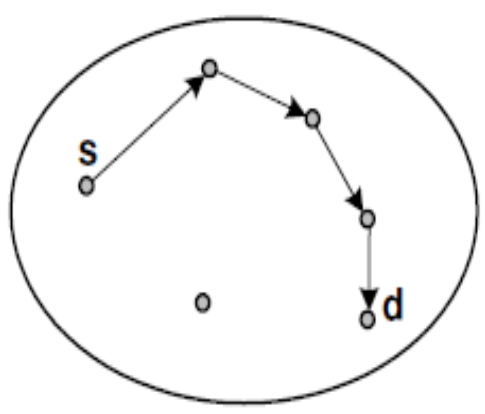

Fig 1.1: multi-hop relaying models

In this thesis we exploit the wireless broadcast property and take the advantages of transmission side diversity to achieve energy savings. 


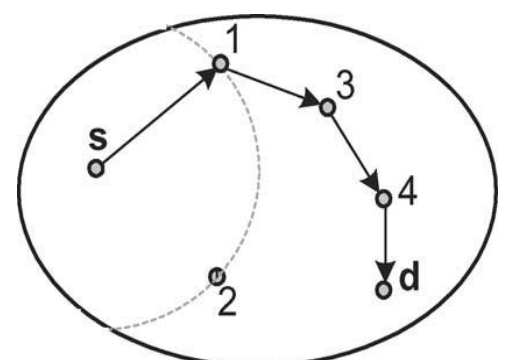

Fig1.2: wireless broadcast advantage

When omni directional antennas are used for communication, the signal transmitted by source node $\mathrm{s}$ is received by all nodes for example here is nodes are 1 and 2 within a certain radius is referred to as the wireless broadcast advantage (WBA)[8]. The problem of finding the minimum energy multicast and broadcast tree in a wireless network is studied in [2] and [3]. This problem is shown to be NP-Complete in [4] and [5]. In this dissertation we have assumed each node is equipped with single omni directional antennas allow many nodes can cooperate to each other is transmitting the information from source to other nodes and through this cooperation effect achieve same energy saving as multiple antenna As we refers as the wireless cooperation advantage (WCA) [11].

\subsection{Cooperative Transmission}

Our objective is to take advantage of the wireless broadcast property and the transmission side diversity through cooperation and in addition, consider the channel variation to find routes that reduce the end-to-end energy consumption, outage probability and some minimum end-to-end throughput. Take the simple example of cooperative transmission Figure 1.1exploits the broadcast nature of the wireless medium. An example of cooperative transmission of minimum energy path from node $S$ to node $D$ using dark line is given in Figure1.2, in which we allow the last two predecessor nodes along the path for cooperative transmission to the next hop, i.e., $h o p=\square 2$.

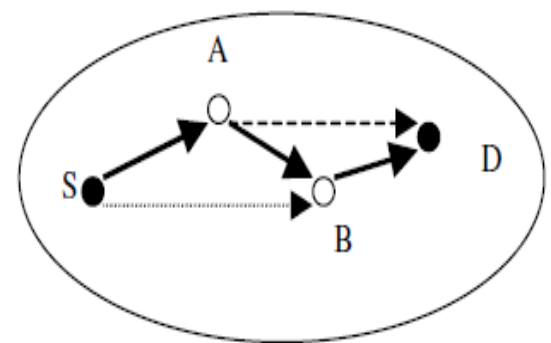

Fig1.3: cooperative transmission

The following steps are for operation of cooperative transmission the first transmission from Node $\mathrm{S}$ and receive Node A, and then B receives the signals transmitted co-operatively by node A and node $\mathrm{S}$.

\subsection{Cooperative Diversity}

Second interesting property of wireless adhoc networks is diversity at the physical layer .The routing protocols point-to-point link and multi-hop link are used to transmit a signal from source node to destination node. Multi-hop link combines the transceiver transmission results that produce increasing SNR or decreasing SNR .SNR fluctuations occur across both frequency and time, also wireless channel variations called as fading, shadowing, and other forms of interference.

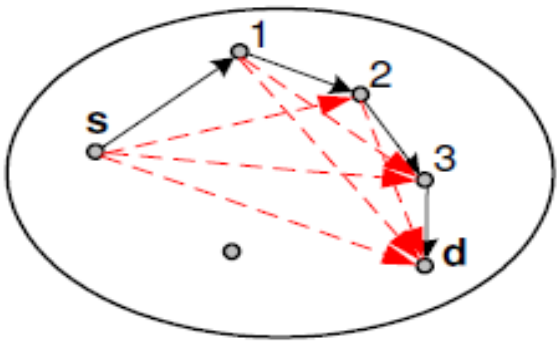

Fig 1.4: cooperative diversity 
Using diversity, the channel interference due to fading is reduced and increases the reliability of the wireless network[4],[7],[9] it can transfer same signal and receiver can detect these multiple copies of sent message correctly by using single Omni directional antenna with space diversity instead of multiple antennas which is costly and occupies more spaces. Then this new technique is known as cooperative diversity. An overview of different transmission diversity techniques is given in [10].the cooperative diversity is achieved by fully decodes and amplifies each information's. In addition to these, the algorithms is evaluate with repetition codes requires low complexity in the terminals by contrast space-time coded cooperative diversity requires more complexity in the terminals

Our goal is to achieve energy saving through jointly cooperation and selection of energy routes with diversity.In order to achieve maximum energy saving by finding optimal route selection and shows probability of error is reduced. The existing literature Khandani et al. [12] they formulate the energy consumption in a static cooperative wireless network shows the maximum energy saving of 50\% compare with non cooperative scheme, the energy savings achieved through cooperation, by using point to point scheme the heuristic algorithms to find energy efficient routes and also consider cooperative Multiple-Input Single-Output (MISO) technique for data transmission and energy savings can be achieved using cooperative routing techniques. In this static network the transmitting nodes must know about channel state information hence nodes are cooperatively beamforming it requires synchronization [15] which is difficult in mobile adhoc network. Zhang et al. [13] extend Khandani's work to a multi-source multi-destination network. The power allocation that minimizes the outage probability with cooperative diversity system was derived in [14]. Our proposed coded cooperative transmission encode the information by using simple repetition code and detect at the receiver, the channel is fully characterized by the channel gain $\mathrm{h}$. The channel gain capture the effects of asynchronism, multipath fading, shadowing and pathloss and it is inversely proportional to $d_{i j}{ }^{\alpha}$. It shows maximum saving of more than $80 \%$ by using coded cooperative transmission than existing non cooperative transmission.

\section{4: Summarization of our dissertation}

1) We formulate the energy optimal cooperative routing between two nodes in fading environment subject to a constraint each node transmission power.

2) We formulate the power allocation for a cooperative diversity with random location of nodes where only mean channel gain is available at the transmitters and are exponentially distribute

3) We develop optimal static routing algorithms to find minimum-energy routes in a network. we derive a Opportunistic cooperative routing as well as develop heuristic cooperative routing algorithms, and evaluate their performance using NS-2.32simulator.

The dissertation is structured as follows. In Section II, we describe our system model and formulate cooperative link. Section III presents our optimal routing formulation and power allocation. Section IV presents Opportunistic cooperative routing Simulation results are presented in Section VI, and Section VII conclusions and future research works are discussed.

\section{System Model}

The wireless network consisting of $\mathrm{N}$ devices are called nodes and it is distributed randomly in an area, where each node has a single omnidirectional antenna. We assume that each node can adjust its transmission power and that multiple nodes can coordinate their transmissions at the physical layer to form a cooperative link. So beamforming is not performed, only rough packet synchronization is required [16].The data throughput, network reliability, link range is increases and reducing fading by using MIMO transmission. We model MIMO transmission as multiple MISO transmission [17].Using MISO we formulate energy consumption in a cooperative MIMO transmission. Let $\mathrm{N}$ be the set of the nodes in the network $\mathrm{N}$, and assume that there are $\mathrm{N}$ $=|\mathrm{N}|$ nodes in the network.

\subsection{Channel model}

We consider a time-slotted wireless channel between source and destination nodes, it is assumed to be fading channel is consist of pair of transmitting node ti, receiving node rj and remaining nodes are serves as a relay nodes (i.e) $\mathrm{N}-2$ nodes in a $\mathrm{N}$ nodes. The source transmits binary symbols. To minimize the effect of noise, fading and reduce the errors of received signal coding technique is used. Encoding of transmitted bits is done before being transmitted and then decoded at receiver. The encoder is using simple repetition code to encode the source output. The decoder is using minimum Hamming distance to detect the transmitted signal. The noise at receiver is assumed to be complex, White Additive Noise with Gaussian distribution of zero mean and without loss of generality the power spectral density $\mathrm{P}_{j}$ is NO .Binary modulation technique is used to modulate the code words. 
The repetition code is used in a coding scheme that they added redundant bits are just a repetition of the original bits across the channel to achieve an error free communication. Repetition coding gets full diversity

Let we assume that the channel is fully characterized by the channel gain $h_{i j}$. The effects of symbol asynchronism, multipath fading, shadowing and path-loss are captured by channel gain and it is inversely proportional to $d_{i j}{ }^{\infty}$

If $\mathbf{x}$ represents the $k$ transmitted bits, then the output of the encoder $\mathbf{c}$ is given by $\mathbf{c}=\mathbf{x G}$

where $\mathbf{G}$ represents the code generator matrix. The transmitted signal will be received at two

\subsection{Cooperative Model}

In this model cooperation at each stage has a collection of multiple-input single-output (MISO) links, where a set of transmitters $T$ cooperatively send data to a set of receivers $R$. Let $h_{i j}, h_{i k}, h_{k j}$ are channel gains between nodes $\mathrm{ij}$, ik and $\mathrm{jk}$ and we assume that they are independent, exponentially distributed random variables with means $m i, j, m i, k$ and $m k, j$, respectively. the receiver node will receive the signal in a two different phases which is transmitted by transmitting node

Phase I: At first time slot the transmitting node i transmit with power $\mathrm{p}_{\mathrm{s} 1}$, and all other relay nodes and receiving node will listen. The transmitted signal $\mathrm{xs}_{1}(\mathrm{t})$ and noise is $\eta_{\mathrm{j}}(\mathrm{t})$ received at $\mathrm{r}_{\mathrm{j}}$, it is assumed to be complex additive white Gaussian with power density $\mathrm{P}_{\mathrm{nj}}$. Then the received signal between $\mathrm{i}$ and $\mathrm{j}$ node is

$y_{i j}=\sqrt{p s_{1_{d_{i j}}^{\text {hij }}}^{{ }_{\text {hij }}}} * \mathrm{xs}_{1}(\mathbf{t})+\eta_{\mathrm{j}}(\mathbf{t})$

Where $h_{i j}$ is channel gain of $i$ and $j$ nodes, $d_{i j}$ is distance between transmitting node $i$ and receiving node $j$ and $\propto$ is the path loss exponents. We assume a non line of sight has the Rayleigh distribution with unit power channel gain magnitude is $\mid$ hij $\mid e^{j \theta i j}$ and $\theta i j$ is the phase.

Similarly the received signal at node $k$ and noise is $\eta_{k}(t)$ received at $r_{k}$,

$y_{i k}=\sqrt{p S_{1_{d_{i k} \mathrm{xx}}}^{\text {hik }}} * \mathrm{xs}_{1}(\mathbf{t})+\boldsymbol{\eta}_{\mathrm{k}}(\mathbf{t})$

If the received signal-to-noise ratio (SNR) exceeds a prescribed decoding threshold $\eta$, the received message can be decoded correctly. Let $\mathrm{D}$ denote the decoded set that includes all the nodes whose received message can be decoded properly.

Phase II: At second time slot the relay node $\mathrm{k}$ transmit signal $\mathrm{xs}_{2}(\mathrm{t})$ to destination node $\mathrm{j}$ with power $\mathrm{pj} \in$ D. $p_{s 2}$ is the power of source node in phase II, and noise is $\eta_{j}(t)$ received at $r_{j}$, it is assumed to be complex additive white Gaussian with power density $P_{n j}$. Then the received signal between $k$ and $j$ node is

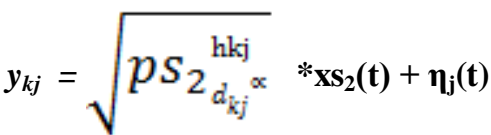

All the decoded nodes, as well as the source node, re-encode the message and transmit with the assigned power. The original transmitted signal combines [18] both the signal received from source and the signal received from relay

$\mathbf{Y}=\left[\mathbf{y}_{\mathbf{i j}}, \mathbf{y}_{\mathbf{k j}}\right]$

Power allocation vectors $\mathrm{p}$, where ps1,ps2 are the power allocated to transmitter at phases I and II respectively. The total transmitted power is $\mathrm{P}_{\mathrm{T}}=\mathrm{Ps}_{1}+\mathrm{Ps}_{2}$ and we have snr at receiver $\mathrm{j} \varepsilon \mathrm{R}$ is

$s n r_{i j}=\frac{\left|h_{i j}\right|^{2}}{d_{i j}{ }^{\alpha}} * \frac{P T}{P \eta j}+\sum_{k \in D} \mathrm{p}_{\mathrm{k}} * \mathrm{~h}_{\mathrm{j}, \mathrm{k}}$

\subsection{Routing Model}

A K-hop cooperative route 1 is a sequence of links ' $\mathrm{k}$ ' is $\left\{1_{1} \ldots \ldots .1_{k}\right\}$, where each link is formed between transmitting node $T_{k}$ and receiving node $r_{k}$ is $l_{k}=\left(t_{k}, r_{k}\right)$ using two stage cooperative transmission. Our objectives to find the route that minimize the end-end transmission power. 
The energy efficient routing can be formulated as follows

$$
\min \sum_{\mathrm{lk \in l}} \mathrm{LC}(\mathrm{tk}, \mathrm{rk})
$$

\subsection{Link Cost Formulation}

Our objective is to determine power allocation for successful transmission from a set of transmitting nodes $S=\left\{\mathrm{s}_{1}, \mathrm{~s}_{2} \ldots . \mathrm{s}_{\mathrm{n}}\right\}$ to a set of terminating nodes $\mathrm{T}=\left\{\mathrm{t}_{1}, \mathrm{t}_{2} \ldots \mathrm{t}_{\mathrm{m}}\right\}$. The link cost can be described in different forms are as follow.

\subsubsection{The point-to-point link}

It is a one-one communication between transmitter $S=\{s 1\}$ and terminator $T=\{t 1\}$ (i.e) $n=1, m=1$ $\operatorname{cost} \mathrm{LC}(s 1, t 1)$.For our simplification let us assume the channel gain is one and that the encoded in a signal has unit power and magnitude of the signal is multiplying a scaling factor $\mathrm{w}$, Then the total transmitted power is $\mathrm{w}^{2}$ is given by

$\mathbf{L C}(s 1, t 1)=\widehat{P T}=\frac{\text { SNRmin } * \text { p } \eta \mathbf{j}}{\alpha^{2}}$

2.4.2 Point-to-Multipoint Link

In this case, $\mathrm{n}=1, \mathrm{~m}>1, \mathrm{~S}=\left\{\mathrm{s}_{1}\right\}, \& \mathrm{~T}=\left\{\mathrm{t}_{1}, \mathrm{t}_{2}, . \mathrm{t}_{\mathrm{m}}\right\} ; \mathrm{m}$ simultaneous SNR constraints must be satisfied at the receivers. The signal transmitted by the source node $s_{1}$ and it is received by all nodes within radius proportional to transmission power is called as broad cast link. Thus, the minimum power required for transmission $\mathrm{LC}(s 1$, $T)$, is given by

$\mathrm{LC}(s, T)=\max \left\{\mathrm{LC}\left(s_{1}, t_{1}\right), \mathrm{LC}\left(s_{1}, t_{2}\right), \ldots, \mathrm{LC}\left(s_{1}, t_{m}\right)\right\}$

\subsubsection{Multipoint-to-Point Link}

In this case, $\mathrm{n}>1, \mathrm{~m}=1, \mathrm{~S}=\left\{\mathrm{s}_{1}, \mathrm{~s}_{2} \ldots \mathrm{s}_{\mathrm{n}}\right\}, \& \mathrm{~T}=\{\mathrm{t} 1\}$. Here multiple nodes cooperate to transmit the same information to a single receiver node is called as cooperative link. Assume the channel gain is known at the transmitter, $h_{j}$ is the vector of channel gains between transmitting nodes in $T$ and a receiver $r_{j} \in R$ and vector $\mathrm{w}$ as the power scaling factor for nodes in $\mathrm{T}$ and dij as the distance between nodes $I$ and $\mathrm{j}, \mathrm{h}_{\mathrm{ij}}$ is the channel gain between $t_{i}$ and receive $r_{j}$.

In this case at the receiver the signals are add up, and complete decoding is possible as long as the received SNR is above the minimum threshold $\mathrm{SNR}_{\min }$ for all nodes in $\mathrm{R}$. Now let us consider the two vectors then the received signal at receiver $r_{j}$ is

$\mathbf{Y}_{\mathbf{j}}=\quad\left(\frac{h_{i j}}{d_{i j}^{\alpha}}\right)^{\mathbf{T}} * \mathbf{w}+\eta \mathbf{j}$

$$
\text { Where } \mathbf{w}=\left[\begin{array}{c}
w_{1} \\
w_{2} \\
\cdot \\
\cdot \\
w_{m}
\end{array}\right] \quad \frac{h_{j}}{d_{i j}^{\alpha}}=\left[\begin{array}{c}
\frac{h_{1 j}}{d_{1 j}^{\alpha c}} \\
\frac{h_{2 j}}{d_{12}^{\alpha c}} \\
\cdot \\
\cdot \\
\frac{h_{m j}}{d_{m j}^{\infty}} .
\end{array}\right]
$$

The power allocation problem for this case is simply

$$
\min \sum_{t i \in T} w_{i}^{2}
$$

$$
\text { s.t. w.r.t } \mathbf{w}^{\mathrm{T}}\left(\frac{\mathrm{h}_{\mathrm{j}}}{d_{i j}^{\alpha}}\right)^{*} \geq \sqrt{\operatorname{SNRmin} * \mathrm{p} \eta *}
$$

The optimization problem can be solved by using the Lagrangian multiplier techniques .The resulting optimal allocation for each node $i$ is given by 
$\widehat{W l}=\frac{\left(\frac{h_{i j}^{*}}{d_{i j}^{\alpha}}\right)}{\sum_{i=1}^{T}\left(\frac{h_{j}}{d_{i j}^{\alpha}}\right) *}$

The resulting cooperative link cost $\mathrm{LC}(S, t 1)$, defined as the optimal total power, is, therefore, given by

$$
\mathbf{L C}(\mathbf{s , t} \mathbf{1})=\sum_{t i \in T} w_{i}^{2}=\frac{\operatorname{SNRmin} * \mathrm{p} \eta *}{\sum_{t i \in T}\left(\frac{h_{i j}^{*}}{d_{i j}^{\alpha}}\right)^{2}}
$$

The above equation can be written in terms of the point-to-point link costs between all the source nodes and destination nodes, given by

$\mathbf{L C}(\mathbf{s}, \mathbf{t} 1)=$ 1

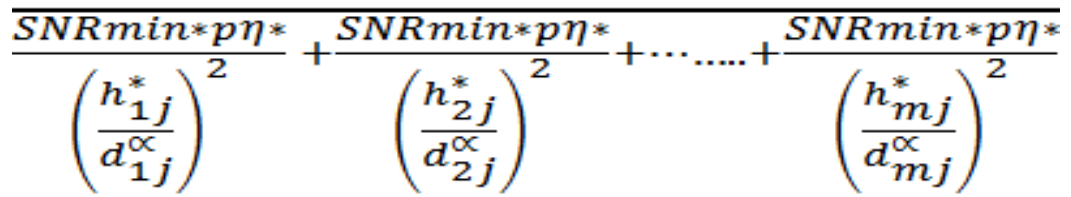

We can conclude that the transmitted signal is proportional to path loss attenuation therefore all nodes in the reliable set cooperate to send the information to a single receiver.Also the cooperative cost is smaller than each point-to-point cost. Hence it proves that always cooperative transmission saving more energy by taking the advantages of WCA.

\section{Optimal Cooperative Routing}

In this section, we develop optimal cooperative routing algorithms and thus find the least cost route from a source node $\mathrm{s}$ to a destination node $\mathrm{d}$ in an arbitrary wireless network. It is multi-hop in nature and selects a cooperative link in every time slot. Let $T_{k}, R_{k}$ are the transmitting and receiving sets in every time slot $\mathrm{k}$. Starting from the source node, the initial transmitting set, T0, is simply $\{\mathrm{s}\}$, and a route is found as soon as the receiving set at some time-slot $\mathrm{k}$ contains the destination node $\mathrm{d}$. Considering the transmitting and receiving sets in previous time-slots, the transmitting set in time slot $k+1$ can be defined in three different ways

\section{3,1. Optimal Cooperative}

From before transmissions, nodes that have data cooperate in the next transmission. The decision variable at the $k$ th stage, denoted by $U_{k}$, determines the set of nodes that will be added to the reliable set in the next transmission slot.

$$
\mathrm{S}_{\mathrm{k}+1}=\mathrm{S}_{\mathrm{k}} \mathrm{UU} \mathrm{U}_{\mathrm{k}} \quad \mathrm{k}=1,2,3 \ldots \ldots
$$

\subsection{Selective Cooperative}

Nodes that are having data from the previous transmission cooperate in that select one best path which is having less outage.

\subsection{Limited Cooperative:}

Only the receiving nodes in previous time-slot cooperate in the next transmission. In this case, the transmitting set in time-slot $\mathrm{k}+1$ is given by.

$$
\mathrm{S}_{\mathrm{k}+1}=\mathbf{R}_{\mathrm{k}} \quad \mathrm{k}=0,1 \ldots \ldots
$$

\subsection{Outage behavior}

In this section, we are discussing the problem of route reliability in wireless networks. Our analysis starts at the reliability of non- cooperative communication and also how the reliability depends on the channel state and distance between the two nodes. Once we develop a result for non cooperative communication, we extend into cooperative communication network. In a network setting, we find the best path between source $S$ 
and destination D for that we first define and analyze the reliability for fixed route and then our proposed algorithm. By taking the advantages of wireless broadcast property the reliability is improved with the idea of diversity.

\subsubsection{Non-Cooperative Transmission.}

Compute the outage probability of a system without cooperative diversity. Consider non-cooperative transmission from source $\mathrm{s}$ to destination $\mathrm{d}$. In this case, the mutual information random variable between source and destination in bits per channel, and as a function of the fading coefficient $\mathrm{h}_{\mathrm{ij}} / \mathrm{d}_{\mathrm{ij}}{ }^{\alpha}$ satisfies Cover and Thomas, 1991; Telatar, 1999

Let the propagations model as consider path loss, Rayleigh fading, Shadow. The wireless link between the nodes $i$ and $j$ is $h_{i j} / d_{i j}{ }^{\alpha}$ Where $d_{i j}$ is the distance between nodes $i$ and $j, h_{i j}$ represents channel gain it captures the effect of channel fading, $\alpha$ is the path loss exponents between the range 2 and 4 .The mutual information as a function of the fading coefficients become a random variables

$$
\mathbf{I}_{\mathrm{NC}} \leq \log \left(1+\frac{\left|\frac{h i j j}{d i j \alpha}\right|^{2} P_{s}}{N o}\right)
$$

Most important parameter in communication system is outage probability or probability of error. An error occurs due to either channel is in outage or decoding error. When the probability of error is 0 the channel is not in outage otherwise outage probability is 1

$\mathbf{P}_{\text {Error }} \approx \mathbf{P}$ (outage)

The outage probability is then the probability that a mutual information falls below fixed rate R chosen a priori. The outage probability for rate R, in bits per channel use, is then given by Ozarow et al., 1994

$$
\mathbf{P}_{\mathrm{NC}} \text { out }=\operatorname{Pr}\left[\mathbf{I}_{\mathrm{NC}} \leq \mathbf{R}\right]=\operatorname{Pr}\left[\left|\frac{h i j}{d i j^{\alpha}}\right|^{2} \leq \frac{2^{R^{2}}-1}{\left(P_{S} / N_{0}\right)}\right]
$$

Without lose of generality the outage probability is given by

$$
\mathbf{P}(\text { Outage })=\operatorname{Pr}\left[\left|\frac{h i j}{d i j^{\alpha}}\right|^{2}<\frac{2^{R}-1}{\left(P_{S} / N 0\right)}\right]
$$

To simplify subsequent derivation, define the function for outage and success as follow;

$$
\begin{array}{lll}
\mathbf{1}_{\text {Outage }}= & \{\mathbf{1} & \text { if }\left|\frac{h i j}{d i j^{\alpha}}\right|^{2} *\left(P_{s} / N 0\right)<\mathbf{1} \\
& \{\mathbf{0} & \text { else and }
\end{array}
$$

\subsubsection{Cooperative Transmission}

Outage results for cooperative transmission are an extension of non cooperative transmission. The simplest amplify-and-forward algorithm for a single source and relay produces an equivalent one-input, twooutput conditional complex Gaussian noise channel with different noise levels in the outputs. As Laneman et al.,2004 details, the mutual information random variable is

$\mathrm{I}_{\mathrm{AF}}=\left(1+2 \frac{\left|\frac{h_{i j}}{d_{i j}^{\alpha}}\right|^{2} P s}{N o}+f\left(2 \frac{\left|\frac{h_{i k}}{d_{i k}^{\alpha}}\right|^{2} P s}{N o}, 2 \frac{\left|\frac{h_{k j}}{d_{k j}^{c}}\right|^{2} P r}{N o}\right)\right)$

For the simplest decode-and-forward algorithm with repetition coding, the mutual information random variable is

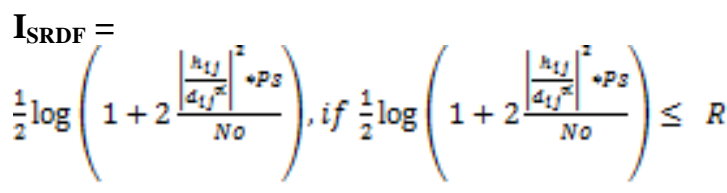




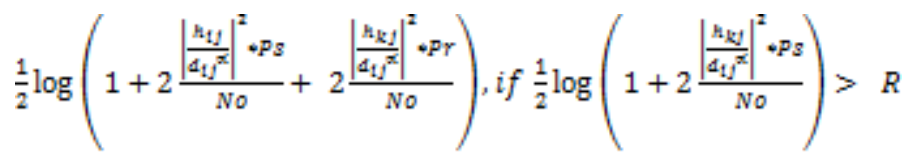

Assuming the assigned bandwidth is same for both source and relay nodes, outage probability (23) in terms of data rate, distances and transmission power can be further expressed as

$\mathbf{P}_{\mathrm{C}}{ }^{\text {out }}=\mathbf{1} / \mathbf{2} \mathbf{d}_{\mathrm{ij}}{ }^{\alpha}\left(\mathbf{d}_{\mathrm{ik}}{ }^{\alpha}+\mathbf{P}_{\mathrm{S}} / \mathbf{P}_{\mathbf{r}} \mathbf{d}_{\mathrm{kj}}{ }^{\alpha}\right) \frac{\left(2^{2 R}-1\right)^{2} \quad *(N o R B)^{2}}{P_{S}^{2}}$

\subsection{Optimal Link Selection}

At each step of routing we should choose $\mathrm{R}$ from all the nodes so that the end-to-end power consumption is minimized.. Let $\mathrm{P}(\mathrm{T})$ denote the total transmission power to reach the destination from transmitting set $\mathrm{T}$ using multi-hop cooperative transmissions. Then, $\mathrm{R}$ is implicitly given by the following optimization problem

\section{Cooperative Routing Algorithm}

An iterative implementation of the routing algorithm works in rounds. Let $\mathrm{h}$ denote the round number, and augment all routing related variables with $\mathrm{h}, e . g ., \mathrm{P}^{\mathrm{h}}(\mathrm{T})$ denotes the routing cost from $\mathrm{T}$ to the destination in round $\mathrm{h}$. Routing variables are updated in each round as follows.

$$
\mathrm{P}^{\mathrm{h}+1}(\mathrm{~T})=\min \left(\left\{\sum_{k} \mathrm{LC}\left(\mathrm{S}_{\mathrm{k}}, \mathrm{U}_{\mathrm{k}}\right)+\mathrm{P}_{\mathrm{C}}{ }^{\text {out }}{ }_{+} \text {available } \mathrm{B}, \mathrm{w}+\mathrm{SNR}_{\mathrm{ij}}\right\}\right) / 4
$$

\section{Opportunistic Cooperative Algorithm}

In the proposed algorithm Opportunistic Minimum Energy Cooperative transmission Shortest Path Algorithms (OMCTSP) the routing protocol AODV is to be modified to implement the routing. In that every step of cooperative routing, all nodes can be overhearing when source nodes sending the route request pkt. After the

transmission to the next node along the non-cooperative shortest path all the nodes that are not in outage, available Bandwidth, size of the packet, residual power available, number of hops will be added to the transmitting set for the next step of the routing. Steps to forward the packet using OMCTSP

1. First modify the MAC layer protocol 802.11 to include SNR calculation

2. Next modify the routing protocol AODV to implement the routing algorithm proposed.

3. Network is deployed with defined no of nodes with specified initial energy

4. SNR value is estimated based on receiving power and distance, while receiving the hello message and SNR value is stored in snr

5. Unit variance model estimates expected value for channel gain for each slot index. Once the square of expected value is 1 then outage probability is estimated.

6. Every node goes for calculating available bandwidth.

7. The number of hops for each path is also calculated.

8. The source node when sending the route request pkt, will include

outage probability, residual power available and available bandwidth, sizeofthepkt.

9 .Intermediate nodes updates the link cost based on point to point connection, and push into request packet along with available bandwidth information.

10.All the forwarding nodes will calculate SNR value of its received pkt, its available bandwidth, residual energy. These values with number of hops are also included in the route request pkt.

11. When the destination node receives the route request packet, it selects the best path based on four parameters.

12. Generate route reply msg to source nod in the reverse path.

13. Once a best path is selected, the destination node sends a route reply pkt to the source node.

14.This calculation goes on for all the pkts forwarded towards the receiver. So that whenever the receiver finds a better path, it discards the old path and picks up the new path for data communication.

15. In future diversity techniques also going to add it shows more energy saving and it will reduces the outage probability also 


\section{1 througput}

\section{Result Analysis}

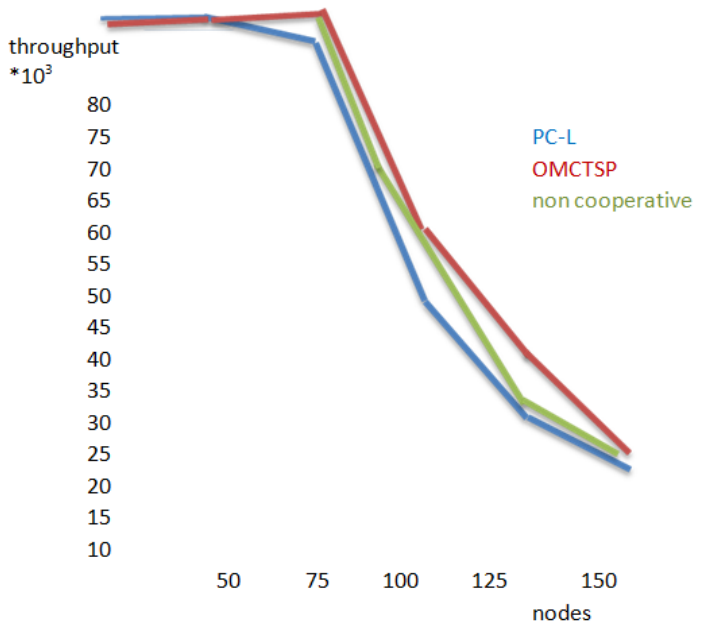

Fig5.1:Shows Nodes Throughput

Throughput: As the number of nodes increases the throughput is comparatively more in the proposed algorithm OMCTSP as compared to other existing algorithms.

\subsection{Packet delivery Ratio:}

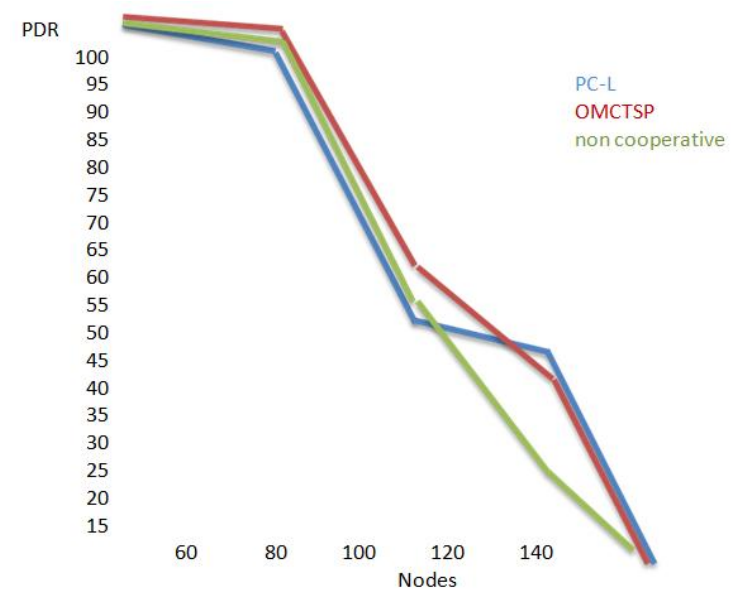

Fig5.2: Shows NodesVs PDR

The packet delivery ratio is fairly larger in the proposed algorithm as compared to the existing non co-operative algorithms in use. The packets delivered is more or less same in PC-L and non co-operative.

\subsection{Average consumed energy}

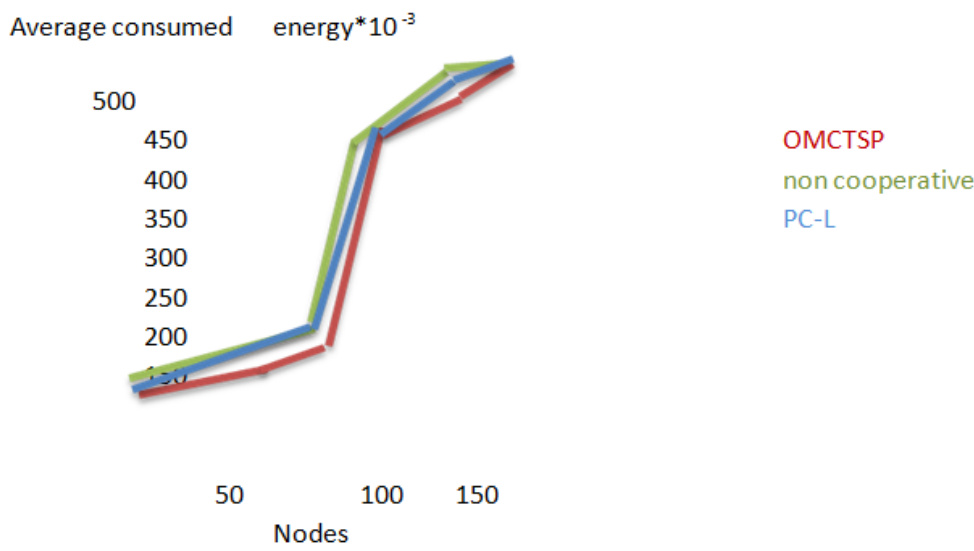

Fig 5.3 Average consumed energy. 
The average consumed energy is comparatively less in the proposed algorithm when the number of nodes are less, but when the number of nodes is more the average consumed energy is fairly equal for the proposed and existing algorithms.

\section{CONCLUSION}

In this paper, we explored jointly problems of cooperative transmission at the physical layer in order to develop energy efficient cooperative Routing algorithms and cooperative diversity [13] for wireless networks. Power is allocated uniformly for all nodes, so beam forming is not required only rough packet synchronization is required. We formulated the optimal routing algorithm that finds energy efficient cooperative routes in polynomial time. Using simulations, we showed that the proposed algorithms are able to find energy efficient routes, and achieve significant energy savings compared to existing routing algorithms. In this paper the proposed algorithm OMCTSP is better as it saves more than $70 \%$ average power compare to existing schemes. In future we can analysis using MIMO scheme with multiflow

\section{Journals Papers}

\section{REFERENCES}

[1] L. M. Feeney and M. Nilsson, "Investigating the energy consumption of a wireless network interface in an ad hoc networking environment," in Proc. INFOCOM 2001, Anchorage, AK, vol. 3, 2007, pp. 1548-1557.

[2] J. H. Chang and L. Tassiulas, "Maximum lifetime routing in wireless sensor networks," IEEE/ACM Transactions on Networking, vol. 12, August 2004.

[3] C. K. Toh, "Maximum battery life routing to support ubiquitous mobile Computing in wireless ad hoc networks," IEEE Communications Magazine, 2001.

[4] J. Laneman and G. Wornell, "Distributed space-time coded protocols for exploiting cooperative diversity in wireless networks," IEEE Trans. Inform. Theory, vol. 49, no. 10, pp. 2415-2425, Oct. 2003

[5] A. Nosratinia, T. Hunter, and A. Hedayat, "Cooperative communication in wireless networks," IEEE Communications Magazine, vol. 42 , no. 10 , pp. $74-80$, Oct. 2004

[6] A. Scaglione, D. L. Goeckel, and J. N. Laneman, "Cooperative communications in mobile ad-hoc networks: Rethinking the link abstraction,"

[7] J. N. Laneman, D. N. Tse, and G. W. Wornell, "Cooperative diversity in wireless networks: Efficient protocols and outage behavior,” IEEE Trans. On Information Theory, vol. 50(12), Dec. 2004. IEEE Signal Processing Magazine, vol. 23, no. 5, pp. 1519, Sep. 2006.

[8] J. E. Wieselthier, G. D. Nguyen, and A. Ephremides, "Algorithms for energy-efficient multicasting in ad hoc wireless networks," Mobile Netw. Appl., vol. 6, no. 3, pp. 251-263, Jun. 2001.

[9] S. M. Alamouti, "A simple transmit diversity technique for wireless communications," IEEE J. Sel. Areas Commun., vol. 16, no. 8, pp. 1451-1458, Oct. 1998 .

[10] R. T. Derryberry, S. D. Gray, D. M. Ionescu, G. Mandyam, and B. Raghothaman, "Transmit diversity in 3G CDMA systems," IEEE Commun. Mag., vol. 40, no. 4, pp. 68-75, Apr. 2002

[11] T. Yung-Szu and G. J. Pottie, "Coherent cooperative transmission from multiple adjacent antennas to a distant stationary antenna through AWGN channels,” in Proc. IEEE 55th Veh. Technol. Conf., 2002, vol. 1, pp. 130-134.

[12] A. Khandani, J. Abounadi, E. Modiano, and L. Zheng, "Cooperative routing in static wireless networks," IEEE Transactions on Wireless Communications, vol. 55, no. 11, pp. 2185-2192, Nov. 2007.

[13] J. Zhang and Q. Zhang, "Cooperative routing in multi-source multi-destination multi-hop wireless networks," in Proc.IEEE Infocom, Phoenix, USA, Apr. 2008, pp. 2369-2377.

[14] M. O. Hasna and M. Alouini, "Optimal power allocation for relayed transmissions over Rayleigh-fading channels," in IEEE Trans. Wireless Commun., vol. 3, no. 6, pp. 1999-2004, Nov. 2004

[15] R. Mudumbai, D. R. B. III, U. Madhow, and H. V. Poor, "Distributed transmit beamforming: Challenges and recent progress," IEEE Communications Magazine, vol. 47, no. 2, pp. 102-110, Feb. 2009.

[16] S. Wei, D. L. Goeckel, and M. C. Valenti, "Asynchronous cooperative diversity," IEEE Trans. Wireless Commun., vol. 5, no. 6, pp. 1547-1557, Jun. 2006.

[17] Y. Lang, C. Bockelmann, D. Wubben, A. Dekorsy, and M. Sollner, “A novel resource allocation strategy for distributed MIMO multi-hop multi-commodity communications," in Proc. ITG Workshop on Smart Antennas, Vienna, Austria, Feb. 2008, pp. 125132.

[19] Thomas M. Cover and Abbas A. El Gamal. Capacity theorems for the relay channel.IEEE Trans. Inform. Theory, 25(5):572 \{584, September 1979

\section{Thesis}

[18] kamal khatir." Coded cooperative communications," thesis of Master of Science in Electrical Engineering Blekinge Institute of Technology Karlskrona, Sweden, September 2007

[20] J.N.Laneman,'Cooperative Diversity in Wireless Network;Algorithms and Architectures,"Ph.D. thesis ,Massachuseets Institute of Technology,Cambridge,MA,August 2002.

[21] I. E. Telatar, ICapacity of Multi-Antenna Gaussian Channels," European Trans. on Telecomm., vol. 10, no. 6, pp. 585\{596, Nov.Dec. 1999. 